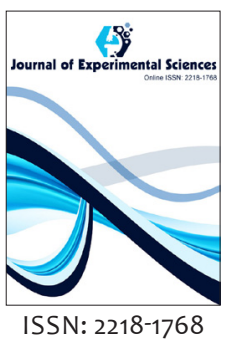

Received: September 17, 2020 Accepted: November 20, 2020 Published: November 26, 2020

*Corresponding author: Maqsudullah Mujtahid, E-mail: mmujtahid61@gmail. com

\section{Blood glucose regulating hormones and their physiological and biochemical roles}

\author{
Maqsudullah Mujtahid* \\ Department of Chemistry, Faculty of Education, Takhar University, Taleqan, Afghanistan
}

\begin{abstract}
Regulation and coordination of different body organs is important in multicellular organisms. Hormones and the nervous system participate in this synchronization. Hormones are the compounds and chemical messengers produced by cells, glands, or special tissues called the endocrine system and released into the bloodstream. In addition to the hormones, blood is consist of proteins, sugars, lipids, vitamins, etc. Blood sugar is glucose which produces energy in the body. Regular blood glucose levels vary at different ages and exceed 120-170 mg/ $\mathrm{dl}$. It is harmful and even fatal to increase or decrease it from the normal level, so the concentration of glucose dissolved in plasma was subject to tight regulation and various regulatory mechanisms were developed thereon. Hormones cortisol, glucagon, adrenaline, and glucocorticoids are the major regulators. These substances are tracked regularly to prevent blood glucose from rising and dropping. Insulin consists of hyperglycemia, as well as other hormones. The mechanism by which it affects the metabolism of the food (carbohydrates, lipids, proteins) prevents hypoglycemia in several respects.
\end{abstract}

KEYWORDS: Hormones, Hyperglycemia, Hypoglycemia, Insulin, Glucagon and Glucocorticoids

\section{INTRODUCTION}

The human body is composed of various tissues. Blood is a normal substance that flows through a closed system known as the circulatory system and consists of two parts: plasma and solid. Plasma contains a variety of biomolecules including vitamins, proteins, enzymes, hormones, carbohydrates, lipids, and non-organics. Glucose is a blood variable, also called blood sugar, and occurs at various ages to some degree. Too much decrease in blood sugar causes hypoglycemia, and increased level of blood glucose causes hyperglycemia (sugar), all of which are detrimental. Naturally, the human body actively works to regulate blood glucose levels, eliminating any potential abnormalities. This is done by various physical or chemical factors. Chemical factors control the level of glucose in the blood by hormones. Hormones are chemicals and trace elements that are synthesized by the endocrine system and secreted as chemical messengers to control and metabolize various metabolic interactions. Hormones are effective at very low concentrations and their amount in the blood is calculated in nanograms [5].

Blood glucose levels are regulated by pancreatic hormones (insulin, glucagon), growth hormones, catecholamines (especially adrenaline), adrenal cortex hormones (glucocorticoids), and thyroxine (thyroid hormone). Insulin prevents various forms of hyperglycemia and the remainder from hypoglycemia. The synthesis and secretion of each of these hormones is more or less in different conditions and under the influence of different biological, chemical, physical, internal or external influences. The aim of this review is to elucidate the significance of hormones in the regulation of blood glucose in human body.

\section{Hormones}

In multicellular organisms, the activity of various organs must be regulated and coordinated, this coordination is achieved by hormones and the nervous system. Hormones control the different metabolic processes and catalyze them. Hormones are able to perform specific tasks at very low concentrations and activate certain enzymes. Hormones are called messengers of chemistry. Because they act as biological regulators by affecting enzyme activity or by acting on cellular organs and thus affecting the speed of biochemical intracellular interactions [5]

The word hormone is a Greek word which means mobilizing or triggering. Hormones are specific chemical compounds, which are secreted directly from the cells, glands or tissues called endocrine glands into the bloodstream and transferred to the target tissue (cells, tissues or organs) through the bloodstream. They have some effects on physiology [12]. 
Hormones are mediating molecules which act in different parts of the cells above the secretion site and regulate their activity in particular ways [7]

Our body has two types of glands; one is the protruding glands, the secretions of which are released through ducts like tears, and glacial glands. And other endocrine glands whose secretions are directed toward the blood vessels for transmission to the target tissue, such as the parotid glands, pancreas, thyroid, adrenal glands. [5]

According to Rohani (2003), some endocrine glands, such as pancreas and genital glands, in addition to internal secretions, also have external secretions called mixed glands.

The endocrine system plays a very effective and valuable role in further strengthening coordination between different organs by producing and secreting chemicals (hormones). The total amount of hormones is very small and in the range of $\mathrm{mol} / \mathrm{Li}$ $-10^{-12} 10^{-9}$. But the target cells and tissues are able to identify the relevant hormones from hormones, and other biomolecules and large amounts of other molecules. This ability is largely provided by cellular receptors or protein receptors in cells [12]

Hormones are derived chemically from amino acids, polypeptides, proteins and/or steroids. Steroid hormones including: androgen, progesterone, testosterone, corticosteroids, hormones with amino acids like adrenaline, noradrenaline, thyroxine. Polypeptide hormones; for example, ACTH, ADH, oxytocin, protein hormones; for example, insulin, glucagon, growth hormone, etc.

Some hormones are derived from lipids; such as, respectively, prostaglandins and steroid hormones made of archidonic acid and cholesterol [5].

\section{Blood}

Frootan (2004) states, blood is a tissue attached to the body that has vital functions. In other words, blood is a regular circular tissue consisting of two main parts.

a. Solid part (blood cells);

b. Liquid part (plasma).

Blood cells are the solid part of the blood but the liquid part of the blood is called the blood plasma, which makes up 55\% of the blood. Around $90 \%$ of plasma is water, and $10 \%$ are solids. Proteins, hormones, carbohydrates, vitamins, lipids, antibodies, and enzymes include plasma solids. Plasma solids include inorganic salts (minerals) and electrolytes [3].

\section{Glucose}

Glucose, also known as blood sugar, is mostly of external origin in the human body, and is derived from carbohydrate breakdown. The organs that need it use it, store the excess in the liver and muscles in the form of glycogen and convert it back to glucose when needed. Glucose chemical formula $\left(\mathrm{C}_{6} \mathrm{H}_{12} \mathrm{O}_{6}\right)$ and its molecular weight is 180 . Glucose produces energy in the cells of the decomposed body or, in the process of its metabolism, may be contained in transformed fat or transformed into nitrogen compounds (amino acids). Glucose metabolism is extremely complex and occurs in a number of ways, the most important of which is the Krebs cycle [3].

\section{The Amount of Glucose in the Blood}

Normal blood glucose levels vary at different ages, which are given below:

a. Infants ............ 30-60 mg / dl;

b. Children ............. 40-90 mg / dl;

c. Children under 12 years....... 60-100 mg / dl;

d. Children older than 12 years ......... 60-110 mg / dl

In the elderly, normal values increase after the age of 50 , critical and dangerous values are higher than $400 \mathrm{mg} / \mathrm{dl}$ and less than $50 \mathrm{mg} / \mathrm{dl}[3]$.

Blood sugar is $90-120 \mathrm{mg} / \mathrm{dl}(0.9-1.2 \mathrm{gr} / \mathrm{li})$ and in the blood cells the amount of sugar is about 0.25 per thousand less than plasma, and after taking blood the concentration of both is almost the same.. After eating foods containing carbohydrates or other hydrocarbons, blood sugar rises to $180 \mathrm{mg} / \mathrm{dl}$ and returns to normal after 2-3 hours. But in patients with diabetes it takes about 5-7 hours [4].

Under normal circumstances, only a small amount of glucose appears in the urine, which can be detected by special examinations, but in diabetes mellitus, the glucose level in the blood rises. If it exceeds $180 \mathrm{mg} / \mathrm{dl}$, it appears in the urine called glycosuria [10].

\section{Blood Glucose-controlling Hormones}

Plasma glucose concentrations are highly controlled to decrease glucose, which causes nausea and mental distress in case of further decrease with dizziness, coma, and extreme hypoglycemia, death, and diabetes-causing hyperglycemia; hence, it is important to maintain the normal blood glucose concentration for living organisms, particularly humans, and various regulatory mechanisms have been established for this. The hormones insulin, glucagon, epinephrine, and glucocorticoids (cortisol) are among the most significant regulating factors and mechanisms. Normal normalization of blood glucose to normal requires the combination of the effects of these hormones on the metabolic processes of many tissues of the body, especially the liver, muscles and adipose tissue [8].

In the following cases, blood glucose rises:

a. Diabetes mellitus;

b. Inject adrenaline and thyroxine;

c. In response to acute stresses such as infection, burns, and surgery relative to glucagon secretion;

d. Cushing's syndrome in proportion to elevated blood cortisol;

e. Rising sugar in anesthesia;

f. Chronic renal failure, which causes an increase in glucagon and glucose; 
g. Pancreatitis causes high blood glucose due to damage caused by inflammation and increased glucagon secretion in the blood;

h. Treatment with diuretic spices.

In the following cases, the amount of blood sugar may decrease and cause hypoglycemia:

a. Excessive increase in insulin in membership;

b. Hypothyroid;

c. Thyroid hormones affect the consumption and production of blood glucose; a decrease in these hormones causes a decrease in blood glucose;

d. Shrinkage of the pituitary gland;

e. Pediatric hypoglycemia;

f. In severe liver disease and cirrhosis of the liver;

g. In Addison's disease [3].

Nelson and Cox (2007) state, hormones that control blood sugar levels are divided into two parts.

a. Hormones that prevent high blood sugar; Such as: insulin;

b. Hormones that prevent low blood sugar; Glucagon, cortisol, adrenaline, thyroxine

\section{Insulin}

The presence of insulin in the extract was isolated from the pancreas for the first time in 1921 and its effects were quickly recognized in lowering blood sugar, and after a short time bovine and porcine insulin was used in the treatment of diabetes among humans. Insulin was the first protein known for its hormonal properties; it was the first protein to be completely processed and crystallized to produce the complete amino acid form and sequence; it was the first protein to be synthesized synthetically. However, modern science still does not have much success in identifying how insulin works at the molecular level, and even our knowledge of how it works is less than most other hormones [11].

Insulin is synthesized and secreted by beta cells in the islets of the pancreas. Its chemical structure was discovered by Sanger, the hormone is made of two polypeptide chains $\mathrm{A}$ and $\mathrm{B}$ and 51 amino acids, chain A contains 21, and chain B contains 30 amino acids. These two chains are connected by two disulfuric connections [12].

Insulin is first developed as preproinsulin, then transformed into proinsulin, and finally into insulin. Periproinsulin is formed by the synthesis of 100 amino acids in the reticulum's endoplasmic reticulum membrane-bound polysomes in the beta cells of the Langerhans islets, and is converted to pro-insulin by the loss of 23 amino acids from the end of its chain. Proinsulin is transferred to the corpus luteum from the endoplasmic reticulum, where it is converted to insulin [6].

\section{Insulin Secretion}

An adult secretes about 50 units of insulin per day, with a total insulin content of 200 to 250 units in the pancreas. Insulin synthesis is continuous, but varying factors (glucose concentration) influence its secretion. The insulin secretion limit concentration of glucose is $80-100 \mathrm{mg} / \mathrm{dl}$. We will have the highest insulin secretion at concentrations of 300-500 glucose. Besides glucose, insulin is stimulated by other sugars such as fructose and mannose, amino acids, pancreatic hormones, digestive hormones (gastrin, secretin), and by some drugs. Insulin secretion is inhibited by hypoglycemia under the influence of somatostatin and some drugs such as nicotinic acid. Insulin in the blood lacks a transporter protein and has a half-life of 5-10 minutes, about fifty percent of this hormone is metabolized in the liver and the rest in the kidneys [12].

24 Insulin units are the equivalent of a milligram. It is dangerous if they suddenly release the same 50 units of insulin into the bloodstream. Insulin release within 30-60 seconds is stimulated directly by the glucose [5].

The normal amount of insulin in the blood is 2.1-30.8il / $\mathrm{mi}$ [3].

\section{The Role of Insulin Metabolism}

Insulin has a great effect on the metabolism of carbohydrates, lipids and proteins.

A. The effect of insulin on carbohydrate metabolism: The main effect of this hormone is lowering blood sugar and increasing glycogen. To do this, it works in the following ways:

a. Insulin increases glucose uptake by muscles, adipose tissue, mammary glands and the lens of the eye;

b. Insulin increases glycolysis in muscles and liver by increasing the synthesis of key enzymes in the glycolysis pathway, including phosphofructokinase and pyruvate kinase. Thus, by increasing glucose uptake by glycolysis, it prevents the release of glucose into the blood plasma.

It stimulates glycogenesis in the liver and in the muscles by activating the glycogen synthase hormone. Insulin inhibits glycogenogenesis through the inactivation of phosphorylase glycogen. Insulin inhibits essential enzymes in the pathways to gluconeogenesis. Insulin increases pyruvate oxidation and transforms it to acetyl coenzyme A following the phosphorylation and transformation of the pyruvate enzyme dihydrogenase into active form.

B. The effect of insulin on lipid metabolism: includes lowering the level of fatty acids, blood and increasing stored triglycerides, which acts as follows:

a. Reduction of lipolysis by inhibition of lipase enzymes;

b. Increased synthesis of Triglycerides;

c. Decreased Ketogenesis

C. Effect on protein metabolism: This hormone increases protein synthesis by increasing the uptake of amino acids by cells, affecting gene transcription, regulating the synthesis of certain types of mRNA, and affecting translation at the ribosome level [12].

In summary, the effect of insulin is to convert excess blood glucose into two forms: glycogen storage (in the liver and muscle) and triglycerides (in adipose tissue) [8]. 
Studying the effects of insulin secretion in humans is the most fascinating way of understanding the physiological effects of insulin on the carbohydrate, lipid and protein metabolism. Hyperglycemia is the most important physiological symptom in diabetes which occurs for three reasons:

a. slowing the entry of glucose into different cells;

b. reduction of glucose consumption in different tissues;

c. Increased production of glucose (Gluconeogenesis) by the liver.

d. Insulin Disorders

Diabetes mellitus is a disorder in the synthesis or action of the hormone insulin in the tissue. The target of diabetes mellitus, which means excessive urination.

a. Type I, in which the disease begins and intensifies rapidly in the early years of life;

b. Type II in which the progression of the disease is slow and usually remains unknown for a long time.

Non-consumption of glucose by tissue, breakdown of fat tissue, glucose excretion along with energy expenditure generally cause diabetic appetite and overeating. Such metabolizing changes arising from impaired insulin activity. Incomplete beta-oxidation in the liver of fatty acids, and high production of ketone bodies. The mouth of sugar patients has a strong odor due to the presence of acetone in the blood, if it progresses it induces ketoacidosis [12].

According to Shabazi and Teya (2006), the main symptoms of diabetes are:

a) increase in urine volume (polyuria);

b) excessive thirst;

c) overeating (Polyphagous);

d) Losing weight despite adequate nutrition

\section{Hormones that Increase Blood Glucose Levels}

The hormones glucagon, adrenaline and glucocorticoids (cortisol) increase blood sugar:

A) Glucagon: polypeptide with 29 amino acids synthesized by alpha cells in the islets of the pancreas. The filament is transported inside the plasma without the transporter protein and has a short half-life. Glucagon has physiological anti-insulin effects [12].

Glucose inhibits glucagon secretion; it is not yet clear; does glucose directly or with the help of insulin and growth factor regulate glucagon secretion? A number of other compounds are more effective in glucagon secretion, such as: amino acids, fatty acids, chitosan compounds, digestive hormones, and neurotransmitters. Some of these citrus fruits stimulate insulin and glucagon to secretion. While others on one of the two hormones have specific nerves, glucagon generally has physiological anti-insulin effects.

According to Zahrayee (2011), factors affecting glucagon secretion include: a) Glucose Unlike insulin, a decrease in blood glucose increases glucagon secretion, but an increase in it directly inhibits glucagon secretion;

b) Increased fatty acids inhibit glucagon secretion;

c) An increase in most amino acids, especially from the fetal fetus, causes the rapid secretion of glucagon;

d) Increased calcium concentration is a strong stimulant in the secretion of this hormone;

e) Growth hormone stimulates glucagon secretion;

f) Exercise stimulates the secretion of this hormone;

g) Secretin and somatostatin inhibit its secretion

\section{Physiological and Metabolic Role of Glucagon}

Blood glucose levels drop a few hours after eating carbohydratecontaining foods due to brain and other tissue oxidation of the glucose. It activates glucagon by reducing blood glucose. This hormone increases glucose in the blood by following method:

a) Inhibits glycogen production from glucose, but stimulates the breakdown of hepatic glycogen into glucose;

b) Inhibits the breakdown of glucose in the liver and activates glucose synthesis by Gluconeogenesis;

c) Prevents the oxidation of pyruvic acid in the crepe cycle;

d) By stimulating hepatic glycogen breakdown, by inhibiting glucose uptake in the glycolysis pathway, and by increasing the production of glucose in the liver, the liver can transport glucose into the bloodstream and maintain normal blood glucose levels;

e) Like epinephrine, glucagon induces the breakdown of triglyceride in adipose tissue. Consequently, free fatty acids are released into the bloodstream and transferred as an energy-producing material to the liver and other tissues. Glucose is only reserved for the brain, and thus glucose is used for energy production in tissues other than the brain [8].

The primary glucagon tissue is the liver. This hormone is fast to allow the body to store free glucose energy sources. Glucose also makes ketone compounds more productive

B) Cuticle Amino acids: The dopamine, epinephrine (adrenaline) and light epinephrine (light adrenaline) hormones are called cuticle amino acids, formed by the central part of the adrenal glands [5].

These hormones are among the amino acid derivative hormones which have a chemical structure with a gasoline ring with two groups of hydroxyl and the first amine side chain. Their precursor is the amino acid tyrosine, 80 per cent of the amino acid cuticle is made up of adrenaline [12].

\section{Effects of Catecholamine Metabolism (adrenaline)}

When an animal is exposed to a stressful condition involving increased activity (in the most severe case, battling or running away), the neurotransmitters release adrenaline and dopamine light from the central part of the adrenal gland. Adrenaline primarily affects the muscles, liver and fat tissue:

a) Epinephrine stimulates glucagon secretion and inhibits insulin secretion; 
b) Epinephrine stimulates the movement of fuels and inhibits their storage.

c) Epinephrine stimulates and breaks down glycerol ions in fat tissue and releases free fat acids into the bloodstream. To be transferred to the liver and other tissues for energy production, and glucose remains in the bloodstream for brain consumption [8].

d) Causes hyperglycemia in the liver and muscles by stimulating glycogenosis. The effect of epinephrine on muscle is far greater than that of glucagon;

e) Epinephrine in the liver increases gluconeogenesis [12].

f) Meeting the immediate needs of the brain for glucose, which is the only source of energy for this tissue;

g) Epinephrine prevents the absorption and consumption of glucose by muscles and other organs [11].

C) Glucocorticoids: Over 50 types of hormones are generated by the cortical portion of the adrenal or adrenal glands, all of which have steroid structure. Only a small number of these compounds are secreted in large quantities, and have physiological activity. Glucocorticoids are so named for their important effect on increased concentration of blood. Cortisol is the most essential of those hormones. Cholesterol is the precursor of all those hormones. Both stages of hormonal synthesis occur in the mitochondria and reticulum endoplasmic [2].

Cortisol's effects are very large, including anti-insulin and pro-insulin effects, protein metabolism effects, lipids, water and electrolyte balance effects, and inhibitory effects on inflammatory and allergic interactions [12].

\section{Biochemical and Physiological Role of Glucocorticoids}

These hormones are important to humans, and their lack or excessive secretion contributes to numerous problems that are directly linked to their metabolism [11].

Different forms of stress; cortisol release triggers excitement, pain, bleeding, infection and hypoglycemia. Cortisol provides the fuel needed to cope with stress by working on the muscles, liver and fat tissue [8].

These hormones influence the metabolism of essential citrus fruits in two distinct ways. They have catabolic effects in some tissues and some anabolic effects; for example, it induces glycogen production in the liver, and it has an insulin-like effect. However, it prevents glucose from entering the cells in peripheral tissue, and exerts an anti-insulin effect [12].

Glucocorticoids affect the metabolism of carbohydrates, proteins, lipids, DNA, RNA and minerals [5].

\section{Effect on Carbohydrate Metabolism}

This hormone has an anti-insulin biological effect on the metabolism of carbohydrates, and causes blood sugar to increase and concentrate as follows: a. Reduces glucose uptake by muscles, adipose tissue cells, and lymphatic cells;

b. Stimulates glucose synthesis;

c. By increasing glycogen synthesis, the storage of this vital compound in the liver increases [12].

d. It synthesizes glucose from a protein in the liver and converts it to glycogen. Without removing glucose from the blood and lowering blood glucose [1].

e. Glycogen stored in the liver is used to support the fight response [8].

f. In the liver, in addition to converting amino acids to glucose, they also increase the conversion of carbon monoxide and glucose [5].

g. Deficiency of this hormone and its production is dangerous. Deficiency of these hormones induces illness in Edison, one of whose symptoms is hypoglycemia. Increased secretion of pituitary origin triggers Cushing's disease, one of whose symptoms is an increase in the production of glucose and hyperglycemia [12].

D) Thyroid hormone (thyroxin): thyroid hormone precursors (thyroxine) are glycoproteins containing $70 \%$ of the inactive hormones MIT and DIT and 30\% of the active hormones T3 and $\mathrm{T} 4$, respectively. This protein is cleaved in the hydrolysis cells and the hormones T3 and T4 and blood flow by peptidase and protease enzymes. Hormones T3 and T4 play a very important role in regulating general body metabolism and development, the tissue differentiation process, gene expression and many critical processes (evolution, increase in basic metabolism, increase in oxygen consumption and heat production) [12].

The normal amount of thyroid hormones T3 is $0.7 / 1.49 \mathrm{ng} /$ $\mathrm{ml}$ and $\mathrm{T} 4=4.5 / 12 \mathrm{ng} / \mathrm{ml}$ in the blood [3] .

\section{Biochemical Effect}

Thyroid hormones have a major impact on the metabolism of proteins, sugars, lipids and minerals, these hormones increase the rate of glucose oxidation, stimulate the absorption of olfactory glucose and increase the production of glucose in the liver. Glucose production in the liver causes the hyperglycemia to increase slightly. Also Glucose-6-Phosporase increases liver activity [5].

E) Growth Hormone (GH): A protein with 191 amino acids, molecular weight is 21,500 , which is secreted by the anterior pituitary glands [5].

Major factors such as nervous activity, sleep, stress, pain, exercise, cold, tiredness, surgery, and nerve shock increase growth hormone secretion. The rate of glucose metabolism in the hypothalamus nuclei is the principal factor that regulates this hormone's secretion. Estrogen, dopamine, glucagon, and digestive hormones add to growth hormone secretion [12].

\section{Physiological and Biochemical Role of Growth Hormone}

The presence of this hormone is essential for the normal postnatal growth and carbohydrate, lipid, protein, and mineral 
metabolism. Unlike insulin, the growth hormone saves on glucose uptake and stimulates gluconeogenesis, thus preventing low blood glucose levels [8].

Growth hormone in the muscles and release of fatty acids from the fatty tissue prevents interactions that degrade glucose. Amino acids increase glycogen in the liver by activating (Gluconeogenesis). Growth hormone injection increases blood glucose by saving uptake of glucose into peripheral tissues and increasing interactions with glucose regeneration in the liver [5].

According to research, the normal amount of growth hormone is as follows:

$$
\begin{gathered}
\text { Male }=\text { upto0.8ng/ml } \\
\text { Female }=\text { upto8.0ng/ml }
\end{gathered}
$$

\section{CONCLUSION}

Human blood is completely fluid, composed of two components (plasma and solids). Blood cells make up the solid part but the plasma part contains $90 \%$ water and $10 \%$ other substances including proteins, hormones, fats, vitamins and so on. Blood sugar is glucose, its normal value is $70-120 \mathrm{mg} / \mathrm{dl}$ in adults and varies in different ages and conditions. Normal levels in elderly people rise after age 50 . Blood sugar level over $400 \mathrm{mg} / \mathrm{dl}$, and below $500 \mathrm{mg} / \mathrm{dl}$ is harmful.

The levels of plasma glucose are highly regulated to prevent hypoglycemia, which can lead to discomfort, confusion, and even death. To prevent hypoglycemia which causes diabetes; it is very important to maintain the normal concentration of blood glucose and various regulatory mechanisms have been developed for it. Hormones are important regulators of blood sugar.

Hormones are organic compounds, and endocrine system glands (necrosis, pancreas, adrenaline, sex, thyroid, etc.) are produced and secreted into the blood. Hormones are chemical mediators and messengers that influence physiological and biochemical activities of human body.

The total amount of hormones is very small and affects different body functions. Insulin, glucagon, epinephrine, glucocorticoids, the growth hormone, T4 and T3 are the hormones responsible for controlling blood glucose levels. Such hormones usually regulate to normal blood glucose. Normal momentary regulation of blood sugar requires the combination of these hormones' effects on the metabolic products of many body tissues, particularly the liver, muscles, and adipose tissue.

These hormones prevent blood disorders by different metabolic pathways having a high effect. Insulin protects glucagon, adrenaline, cortisol, T3, T4 and growth hormone from hyperglycemia and hormones by reducing the metabolic impact of carbohydrates, lipids and proteins.

\section{REFERENCES}

1. Anwar, M. A. Device Chemistry. Kabul: Azeem; 2011.

Frootan, M. N. Physiology. Kabul: Laghmani; 2004.

Khushki, S. Z. Blood biochemistry. Kabul: Azeem; 2005.

Mafi, M. A. Chemistry biology. Tehran: Tehran University; 1953.

5. Mehr, A. R. General biochemistry for agriculture and veterinary. Dasht: Haq Shenas; 2006.

6. Murry, R. K., Granner, D. K., Mayes, P. A., \& Rodwell, V. W. Harper's illustrated biochemistry. (S. A. Mehr, Trans.) Tehran: Tabib; 2007.

7. Nasimi, M. T. Human Anatomy and physiology. Kabul: Afghan Times; 2012.

8. Nelson, D. L., \& Cox, M. M. Lehninger principle of biochemistry. (R. Mohammadi, Trans.) Tehran: AlJ; 2007.

9. Rahim, S. R. Medical biochemistry. Kabul: Azeem; 2010.

10. Rohani, S. A. Physiology of the inner nerves and endocrine glands. Tehran: Samt; 2003.

11. Shahbazi, P., \& Teya, N. M. General biochemistry for medical students. Tehran: Tehran University; 2006.

12. Zahrayee, M. Medical biochemistry. Tehran: AlJ; 2011. 